EPJ Web of Conferences 77, 00017 (2014)

DOI: 10.1051 /epjconf / 20147700017

C Owned by the authors, published by EDP Sciences, 2014

\title{
Compact atomic clock prototype based on coherent population trapping
}

\author{
Jean-Marie Danet ${ }^{1, a}$, Olga Kozlova ${ }^{1,2}$, Peter Yun $^{1}$, Stéphane Guérandel ${ }^{1}$ and Emeric de Clercq ${ }^{1}$ \\ ${ }^{1}$ Laboratoire national de métrologie et d'essais - Systèmes de Référence Temps-Espace (LNE-SYRTE), Observatoire de \\ Paris, CNRS UMR 8630, Université Pierre et Marie Curie, 61 Avenue de l'Observatoire, 75014 Paris, France. \\ ${ }^{2}$ present address: Laboratoire Commun de Métrologie LNE-CNAM, 61 rue du Landy, 93210 La Plaine Saint Denis, \\ France.
}

\begin{abstract}
Toward the next generations of compact atomic clocks, clocks based on coherent population trapping (CPT) offer a very interesting alternative. Thanks to CPT, a quantum interfering process, this technology has made a decisive step in the miniaturization direction. Fractional frequency stability of $1.5 \times 10^{-10}$ at $1 \mathrm{~s}$ has been demonstrated in commercial devices of a few $\mathrm{cm}^{3}$. The laboratory prototype presented here intends to explore what could be the ultimate stability of a CPT based device. To do so, an original double- $\Lambda$ optical scheme and a pulsed interrogation have been implemented in order to get a good compromise between contrast and linewidth. A study of two main sources of noise, the relative intensity and the local oscillator (LO) noise, has been performed. By designing simple solutions, it led to a new fractional frequency limitation lower than $4 \times 10^{-13}$ at 1 $\mathrm{s}$ integration. Such a performance proves that such a technology could rival with classical ones as double resonance clocks.
\end{abstract}

\section{Introduction}

Within the high technology domain, many devices need on-board high performance clocks. The most famous example is the global navigation satellite systems (GNSS), where a $1 \mu$ s error of the on-board clock could induce a $300 \mathrm{~m}$ error on the localization on earth. The current stability performances of vapour cell atomic clocks used in the Galileo program are $5 \times 10^{-12}$ at $1 \mathrm{~s}$ [1]. Laboratories are now preparing the next generations with the challenge to reach at least $5 \times 10^{-13}$ at $1 \mathrm{~s}$ as fractional frequency stability. The project presented here describes a compact atomic clock laboratory prototype which uses an innovative atoms interrogation technique called coherent population trapping (CPT). Contrary to the double resonance clock today used in the GPS, the microwave pulses are optically carried. This allows the suppression of the microwave cavity and leads to a high miniaturization potential.

In this paper we will describe a setup built to reach the highest stability. Such a study precedes the compacification process and intends to prove a stability better than $5.10^{-13}$ at $1 \mathrm{~s}$ averaging time, showing a dominant white frequency noise during $10^{4} \mathrm{~s}$. Such performances are required by the next GNSS satellite generation.

The CPT phenomenon is firstly described in section II, its interest and operation requirements are detailed. The next section presents the experimental setup used. The section IV is dedicated to the frequency stability and the signal-tonoise ratio which is the key parameter in a metrological setup, if one wants to improve its performances.

\footnotetext{
${ }^{a}$ Corresponding author: jean-marie.danet@obspm.fr
}

\section{Coherent population trapping in a vapour cell clock: what, why, and how}

\subsection{What is CPT}

CPT was discovered at Pise in 1976, by Alzetta and his colleagues [2]. By irradiating with a multimode laser a sodium vapor cell placed in a magnetic field inhomogeneous along the cell axis, they noticed that the bright fluorescent cell was striped by dark lines in different places. Here the frequency difference separating two Zeeman sublevels was equal to the frequency difference of two laser modes. This has been explained as follow: after a couple of absorption-spontaneous emission cycles, the atoms are placed in a trapped state where they do not absorb light anymore. This state, called dark state, is a linear superposition of two states for which a destructive interference occurs between the transition amplitude probabilities towards a third excited state. Various explanations of such a dark state from different points of view have been reviewed by Arimondo [3]. Let us now consider a setup where the multimode laser is replaced by two single mode ones which are interacting with a three energy level atom. Among those three levels, two are long-lived states in the ground state and one is an excited state, see Fig. 1.

When the first laser is resonant with the transition $\left(3 \rightarrow 3^{\prime}\right)$ and the frequency of the second laser is swept around the second transition $\left(4 \rightarrow 3^{\prime}\right)$, as a first guess, one could wait the atoms absorb more and more light when the second laser frequency gets closer from the resonance. It is exactly what happens until a certain point. As the frequency gets 
close enough to resonance, the absorption process stops suddenly (see Fig. 2), because the probability amplitudes of transitions $3 \rightarrow 3$ ' and $4 \rightarrow 3$ ' enter in phase opposition. This is what is called CPT.

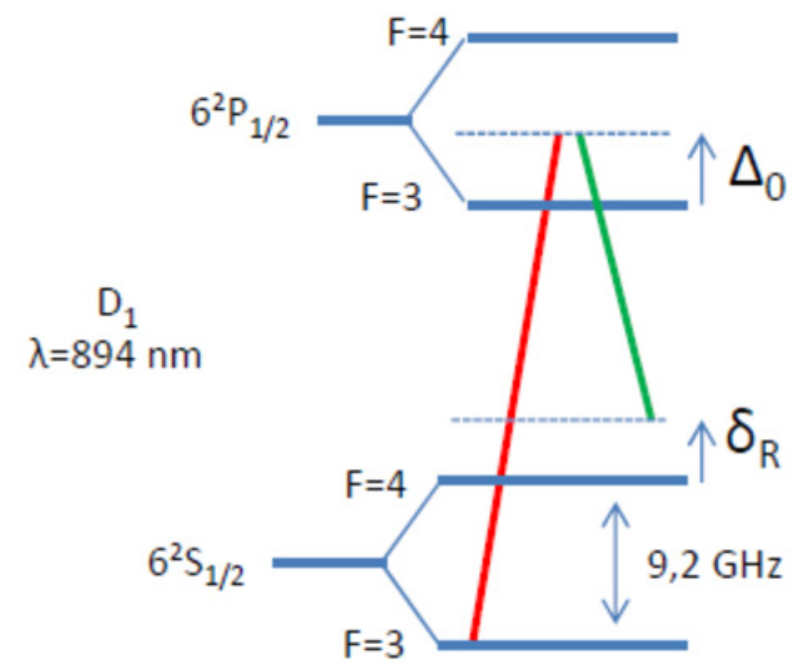

Figure 1. Scheme of the involved three energy levels of the cesium atom. $\Delta_{0}$ is the optical detuning, $\delta_{\mathrm{R}}$ is the Raman detuning.

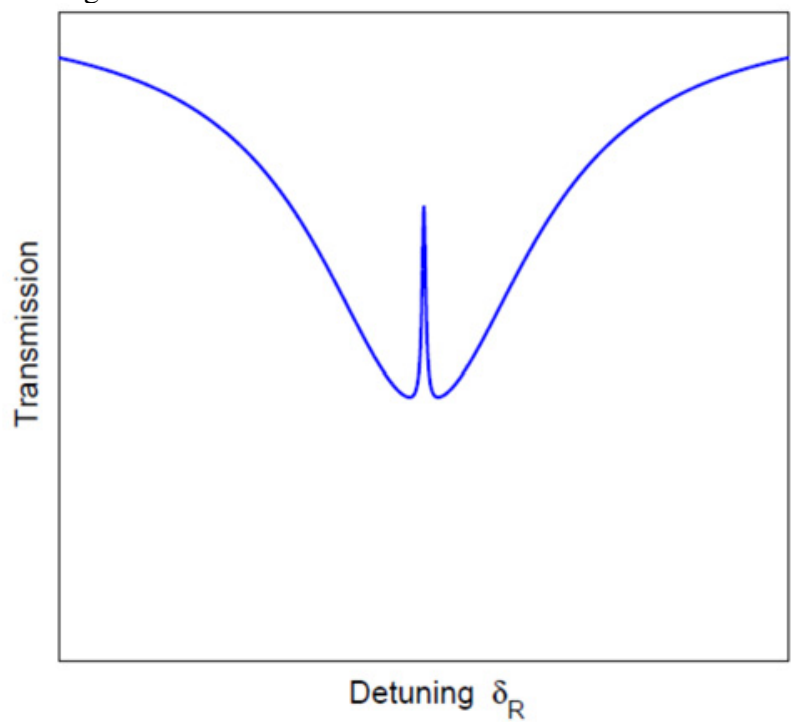

Figure 2. Example of transmission as a function of Raman detuning $\delta_{\mathrm{R}}$. The dark resonance stands out of the optical absorption background. The medium is not completely transparent at resonance because of the absorption of atoms located in other Zeeman sublevels that are not involved in the dark state.

\subsection{Why developing a clock with CPT}

CPT phenomenon has different advantages for a compact clock application. Firstly, it can be used with some of the technology already developed for spatial commercial clocks as the vapour cells or the microwave sources. For such devices, decades of research and exploitation have proved their efficiency and reliability. Secondly its major innovation is to replace the microwave interrogation by an all optical one, enabling highly miniaturized devices. CPT makes possible preparation of the atoms, resonant excitation, and detection of the resonance in the same place and at the same time. The same laser beams are used to create the dark state, and to detect it in the same time by monitoring the transmitted light power. Even if the CPT resonance is created using two optical transitions, its ultimate linewidth is independent of the widths of the optical transitions; it is only related to the lifetime of the coherence between the two ground states. Coherent population trapping is thus a very attractive tool for high resolution spectroscopy, with potential applications to magnetometers and atomic clocks [4-6]. Unlike vapor cell clocks as the widespread rubidium clocks, CPT clocks do not need microwave cavity, since the resonance interrogation is all-optical; the microwave signal is carried by the laser beams. This makes possible miniaturized atomic clocks with a volume of a few tens of $\mathrm{cm}^{3}[7,8]$.

\subsection{How can it be used?}

Let us now guess that one wants to use CPT to build an atomic clock. As in every other atomic clock, the goal is to lock the local oscillator (LO) frequency on the very stable frequency of an atomic transition. The clock signal which is a LO frequency in the microwave range, is then sent to a frequency counter where the frequency signal is converted into a time scale. A simple CPT clock setup is shown in Fig 3.

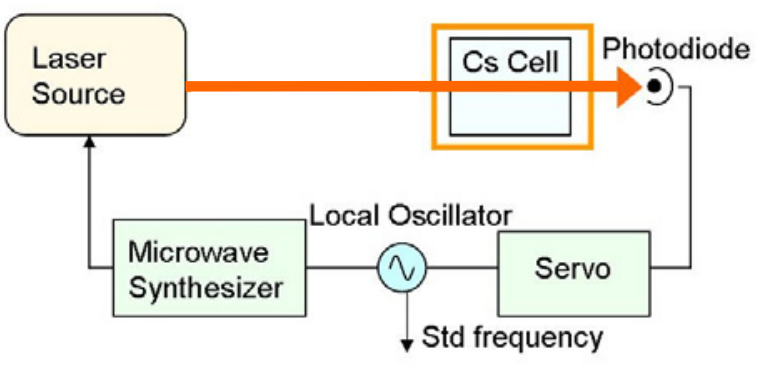

Figure 3 . Basic scheme of a CPT atomic clock.

Alkali-metal atoms properties are well adapted for microwave clocks as $\mathrm{Cs}$ beam clocks and $\mathrm{Rb}$ vapor cell clocks. The CPT phenomenon has been observed on both atomic species. We chose Cs atom for our prototype for some theoretical and practical reasons. Cs atom is far to be a three-level atom, but three levels can be selected by the lasers used. The two laser frequencies are often generated by modulating the frequency or the amplitude of a single laser. This generates many sidebands and makes difficult the analysis of the phenomenon. It is why we chose in a first step using two lasers, with one phase-locked on the other one. This scheme enables more versatility and detailed investigations. The Cs vapor is confined in a glass cell. In order to increase the coherence lifetime which limits the resonance width, a buffer gas is introduced in the cell [9]. Otherwise the width is limited by the short atomic transit time between the cell walls. The Cs-buffers gas collisions slow down the diffusion of Cs atoms towards the walls, but they shift the mean resonance frequency. It is worth to note that the resonance width increases quickly with the laser intensity. Consequently CPT clocks use weak laser intensity of about a few $\mu \mathrm{W} / \mathrm{cm}^{2}$ at the expense of the signal amplitude. This can be circumvented using a Ramsey technique interrogation [10]. When the atomic vapor is irradiated by two laser pulses separated by a time 
$T$ the resonance width scales as $1 /(2 T)$ independently of the laser intensity. Narrow resonances and high signals are then possible [11].

\section{Experimental setup}

The two frequencies are generated by two phase-locked extended cavity diode laser (ECDL). A first control loop is used to lock the master laser to the $\left(F=4-F^{\prime}=4\right)$ hyperfine component of the Cs $D_{1}$ line (wavelength 894 $\mathrm{nm}$ ) by saturated absorption in an auxiliary vapor cell. A second loop is then used to phase locked the $2^{\text {nd }}$ laser to the master laser with a frequency offset tunable around 9.192 $\mathrm{GHz}$. For this purpose, the two beams are superimposed and detected by the fast photodiode PD2, see Fig. 4. The $9.192 \mathrm{GHz}$ beat note is compared in phase to a $9.392 \mathrm{GHz}$ signal issued from a low noise frequency synthesizer. The resulting $200 \mathrm{MHz}$ signal is then used in the phase lock loop (PLL) to lock the slave laser.

The frequency synthesizer [12] is build as follows. A 100 $\mathrm{MHz}$ local oscillator (LO) is phase locked on the $100 \mathrm{MHz}$ signal generated by an H-maser of the laboratory. The LO signal is frequency multiplied to produce a $9400 \mathrm{MHz}$ signal, which is mixed with a $7.4 \mathrm{MHz}$ signal to yield the tunable $9392 \mathrm{MHz}$ signal. The 7.4 MHz signal is produced by a home-made direct digital synthesizer (DDS), whose phase or frequency can be controlled by a computer (PC).

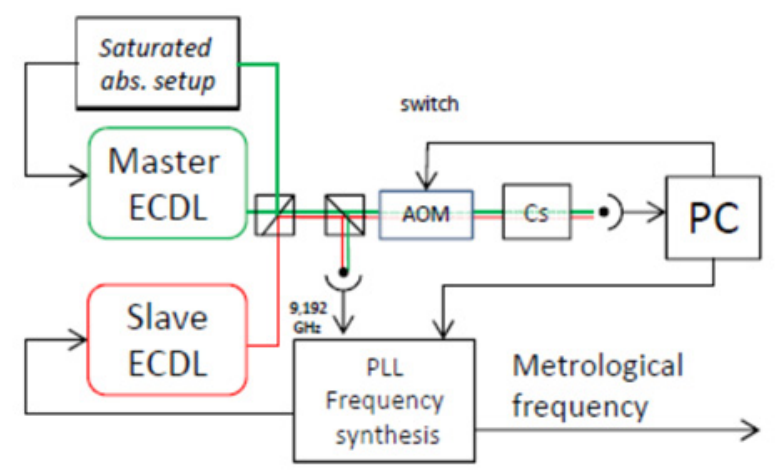

Figure 4 . Experimental setup. Green: Master extended cavity diode laser (ECDL). Red: Slave ECDL locked at $9 \mathrm{GHz}$ from the master frequency using a phase locked loop (PLL). AOM acousto-optic modulator, PC computer. Clock evaluation is made by comparison with an $\mathrm{H}$-maser. Cell : $\mathrm{Ar} / \mathrm{N}_{2}$ mixture.

The two superimposed laser beams are switched on and off by an acousto-optic modulator (AOM) before travelling across the $\mathrm{Cs}$ cell. The $\mathrm{Cs}$ cell, $5 \mathrm{~cm}$ long and 2 $\mathrm{cm}$ diameter, is filled with a buffer gas $\left(\mathrm{N}_{2}-\mathrm{Ar}\right.$ mixture $)$. The cell is temperature controlled to within the $\mathrm{mK}$ level. It is surrounded by a solenoid applying a static axial magnetic field and two magnetic shields. The transmitted power is recorded by a low-noise Si photodiode (PD1). The resulting digitalized signal is processed by a computer (PC) which drives the 7.4 MHz synthesizer as well as the AOM switch.

A digital loop is used in order to lock the synthesizer frequency. To do so, the interrogation frequency (IF) is hold at resonance, and the phase of the synthesizer is square-wave modulated of $\pm \pi / 2$ during the period in the dark. The error signal is computed by the PC which can lock the LO frequency or equivalently the synthesizer frequency. Its frequency is proportional to the frequency difference between the maser and the Cs clock and the Allan variance is computed from its record.

\section{Frequency stability}

The frequency stability of an atomic clock is characterized by its Allan variance. Assuming the stability to be limited by two dominant effects, laser relative intensity noise (RIN) and LO noise, the Allan variance is:

$$
\sigma_{y}^{2}(\tau)=\sigma_{y S N R}^{2}(\tau)+\sigma_{y D i c k}^{2}(\tau)
$$

with $\sigma^{2}$ y SNR the contribution of the RIN to the signal to noise ratio (SNR) and $\sigma_{y}^{2}$ Dick the contribution of the LO phase noise. A complete expression of $\sigma^{2}$ y Dick will be given in Eq (5). Using the detailed expression of $\sigma^{2}$ y SNR the Allan deviation can be written as:

$$
\sigma_{y}^{2}(\tau) \approx\left(\frac{\sigma_{C \text { rin }}}{v_{0} C / \Delta v} \sqrt{\frac{T c}{\tau}}\right)^{2}+\sigma_{y \text { Dick }}^{2}(\tau)
$$

$\sigma_{\text {Crin }}$ is the contribution of the fractional standard deviation of the laser intensity (calculated in Eq (3)), $\Delta v$ the resonance width, $v_{0}$ the resonance frequency, $T_{c}$ the time of an interrogation cycle, and $\tau$ the averaging time, $C$ is the contrast of the resonance defined as the signal amplitude divided by the background. In the following part we will describe the choices that allowed our clock to show a short term stability lower than $5 \times 10^{-13}$ at $1 \mathrm{~s}$.

\subsection{Linewidth $\Delta v$}

Narrower linewidths can be obtained by combining coherent population trapping and a Ramsey interrogation technique. In this case a first laser pulse pumps the atoms in the dark state, which is a steady-state. It is analog to optical pumping, using spontaneous emission process, there is no equivalent of a $\pi / 2$ pulse like in traditional microwave Cs clocks [9]. The second Ramsey pulse must be very short, otherwise the atoms are pumped again in the steady-state, the fringes vanish $[11,12]$. The same pulse is also used for signal detection, by means of fluorescence or absorption. A cycle of operation of a Ramsey CPT clock is then very simple and different from that of traditional clocks. It consists of a long pulse and a short pulse train separated by a delay time $T_{R}$, see Fig. 5 . 


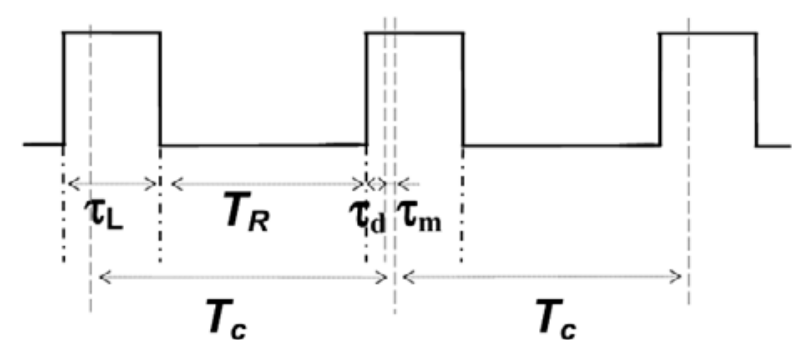

Figure 5 . Time sequence of a CPT Ramsey clock (not to scale). $\tau_{\mathrm{L}}$ is the length of each laser pulse, $T_{R}$ is the free evolution time. The signal is detected after a time $\tau_{\mathrm{d}}$ at the beginning of each pulse, and averaged during a time $\tau_{\mathrm{m}}$. The length of a cycle is $T_{c}$ $=\tau_{\mathrm{L}}-\left(\tau_{\mathrm{d}}+\tau_{\mathrm{m}}\right)+T_{R}+\tau_{\mathrm{d}}+\tau_{\mathrm{m}}=\tau_{\mathrm{L}}+T_{R}$.

In clock operation the Cs vapor is illuminated by a train of laser pulses, of length $\tau_{\mathrm{L}}$ (typically $2 \mathrm{~ms}$ in our set-up), separated by a Ramsey time $T_{R}(4 \mathrm{~ms})$ during which the atoms evolve freely in the dark. At the beginning of each pulse the signal is detected after a time $\tau_{\mathrm{d}}(10 \mu \mathrm{s})$, and averaged during a time $\tau_{\mathrm{m}}(25 \mu \mathrm{s})$, corresponding to the end of each cycle. The atoms are then pumped again in the dark state. The full length of a cycle is $T_{c}=\tau_{\mathrm{L}}+T_{R}$. The obtained Ramsey fringes are shown in Fig. 6.

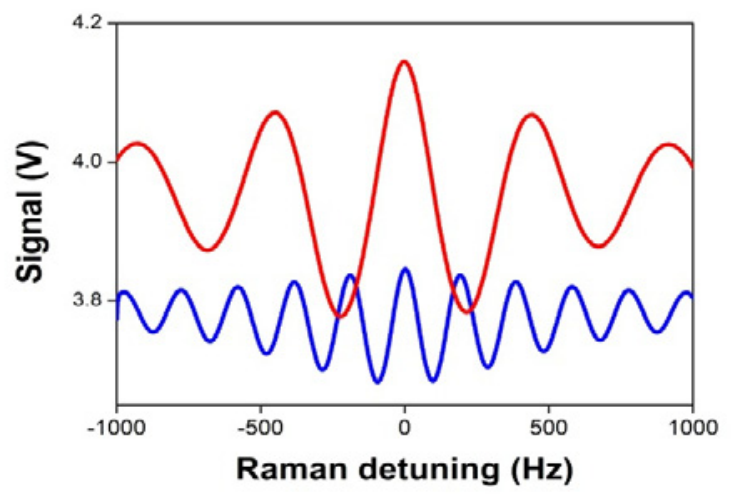

Figure 6. Experimental Ramsey fringes for $2 \mathrm{~ms}$ (upper red curve) and $5 \mathrm{~ms}$ interrogation time (lower blue curve).

\subsection{Contrast}

In most of the CPT setups the lasers are circularly polarized. The atoms are optically pumped in an extreme Zeeman sublevel where they are trapped. For example, for a $\sigma^{+}$polarization the trap will be the Zeeman state $m=+4$. However, if the lasers are linearly and perpendicularly polarized ( $\operatorname{Lin} \perp \operatorname{Lin})$, the excitation scheme is equivalent to $\sigma^{-}$and $\sigma^{+}$polarized light. The trapping level will then be continuously emptied by the $\sigma^{-}$laser beams, see Fig 7 , bottom. The main effect is to have more atoms available on the clock transition $((F=3, m=0)-(F=4, m=0))$ because of its low magnetic sensitivity. $F$ is the hyperfine quantum number and $m$ the magnetic quantum number.
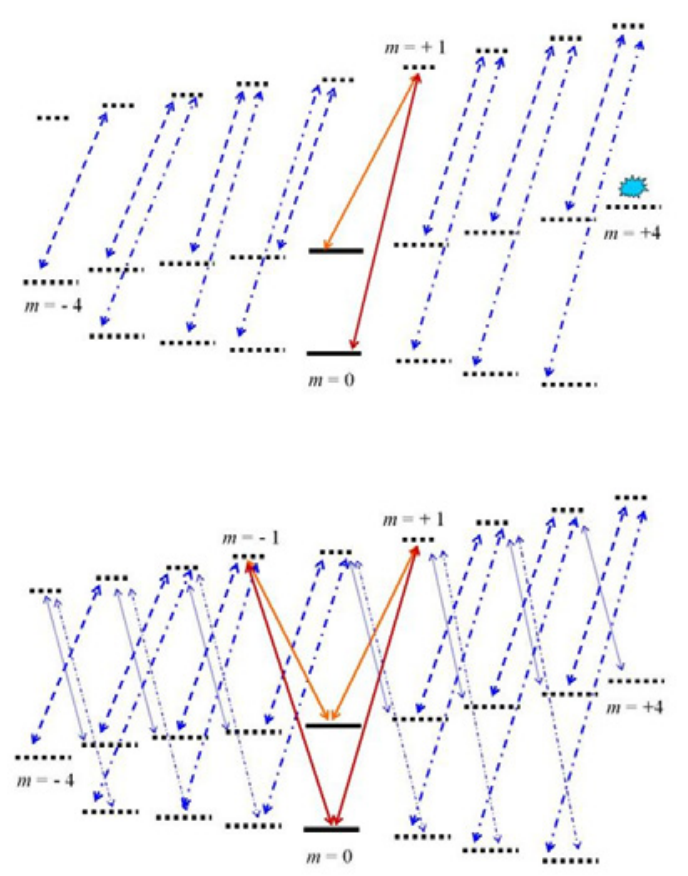

Figure 7 . Cesium excitation scheme: Top: circularly polarized light $\left(\sigma^{+}\right)$, a part of the atoms are pumped in the extreme Zeeman sublevel $(\mathrm{F}=4, \mathrm{~m}=4)$. Bottom: double lambda scheme $\left(\sigma^{+}\right.$and $\sigma^{-}$ ), the atoms are circulating over all the Zeeman sublevels. The dark resonance takes place for the Zeeman level couple for which the frequency difference equals the laser frequency difference. The excited state is $F^{\prime}=4$.

If one compares the two schemes used with a Ramsey interrogation [14], with the same power, the Lin $\perp$ Lin scheme can give $31 \%$ of contrast compared to $2.8 \%$ in the $\sigma^{+}$case.

\subsection{Noise}

\subsubsection{Intensity noise}

A variation of the laser power is responsible from various effects in our CPT clocks. The light shift $[5,9,15]$ which describes the way the atomic frequency is shifted by the laser power contributes at the $10^{-14}$ level to the fractional frequency measured at $1 \mathrm{~s}$, so it doesn't affect the target $10^{-13}$ performance for this clock. However, the laser intensity noise also creates an amplitude noise on the detected signal and then a frequency noise through the LO lock loop. The generation of the error signal is based on the measurement of the difference of signal levels from the right and left side of the central fringe. From this differential detection, where the signals are measured during $\tau_{\mathrm{m}}=25 \mu \mathrm{s}$ (bandwidth $B W=20 \mathrm{kHz}$ ), results a contribution of the RIN noise seen by the clock which can be written [17] :

$$
\sigma_{C r i n}^{2}=\frac{1}{N} \int_{0}^{B W} 2\left(1-\cos (2 \pi f T c) S_{r i n} d f,\right.
$$


where $S_{\text {rin }}$ is the RIN power spectral density, $N$ is the number of cycles over which the signal is averaged. In our working conditions where $N=10$ this noise has to be at the level of $\sigma_{\text {Crin }}=5 \times 10^{-6}\left(5 \times 10^{-5}\right)$ to get a $10^{-13}\left(10^{-12}\right)$ contribution to the total fractional frequency stability. As it is measured at the $2.5 \times 10^{-5}$ level, a study have been undertaken to reduce it.

The first solution, Sol 1 in Fig. 8, was to lock independently both laser powers. To do so an AOM is implemented on each beam trajectory and a beam splitter send $10 \%$ of its 0 order to a photodiode. This signal is compared with a voltage reference and a PI loop acts back on the RF power of the AOM.

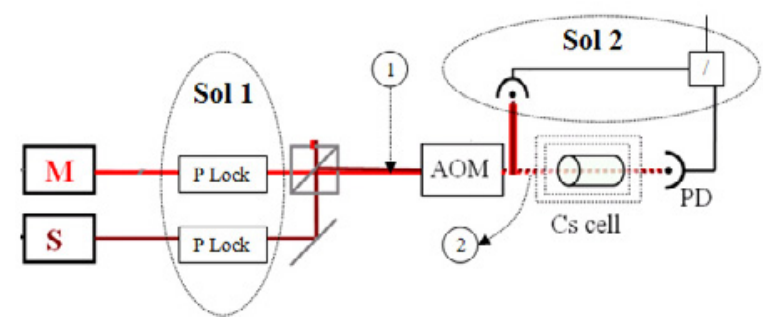

Figure 8. Test to reduce the RIN contribution to the clock frequency stability. Sol1: laser-power locks using an acoustooptical modulator (AOM). Sol2: Normalization of the signal by the measured intensity before the cell.

As shown in Fig 9, for a measurement at point 1 of Fig. 8, this setup reduces the $S_{\text {rin }}$ from $-115 \mathrm{~dB} / \mathrm{Hz}$ (green curve) to $-140 \mathrm{~dB} / \mathrm{Hz}$ (black curve) at the clock modulation frequency, $160 \mathrm{~Hz}$. This level is almost limited by the noise of the voltage reference and the detector noise, here at $-144 \mathrm{~dB} / \mathrm{Hz}$ at $160 \mathrm{~Hz}$, in blue on Fig. 9. Unfortunately, after the switching AOM (point 2 on Fig. 8), a serious degradation is observed (red curve in Fig 9) which limits the performance of this system at $-130 \mathrm{~dB} / \mathrm{Hz}$ at $160 \mathrm{~Hz}$.

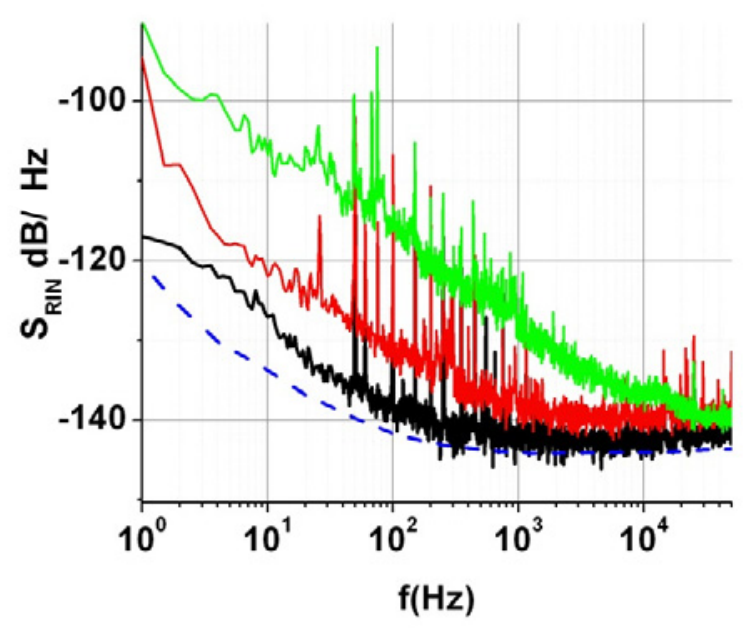

Figure 9. Power spectral density of the RIN in $\mathrm{dB} / \mathrm{Hz}$. Green (upper solid curve): power lock OFF. Black (lower solid curve): power lock ON, measurement before the swtiching AOM. Red (middle solid curve): power lock $\mathrm{ON}$, measurement after the switching AOM. Blue dashed curve: contribution of the voltage reference and photo-detector noise.
The corresponding $\sigma_{\text {Crin }}$ equals $9 \times 10^{-6}$ which contributes to the fractional frequency stability at the $1.9 \times 10^{-13}$ level.

A second way to reduce the RIN is based on the idea that if the intensity noise dominates the noise budget, the fluctuations on the detected signal might be proportional to the intensity fluctuations before the cell (point 2 in Fig. 8). This has been successfully checked on our setup by the following test. The total laser beam intensity $I$ has been modulated with a peak to peak value $\Delta I$ and the corresponding amplitude modulation index $m=\Delta I / I$ measured before and after the cell have been compared. As shown on Fig 10, it appeared that $m$ remain unchanged by the atomic resonator either on or out of resonance, for modulation frequencies between $10 \mathrm{~Hz}$ and $40 \mathrm{kHz}$. Thus in our sensitivity bandwidth which is $80 \mathrm{~Hz}-20 \mathrm{kHz}$, the RIN is the same before and after the cell.

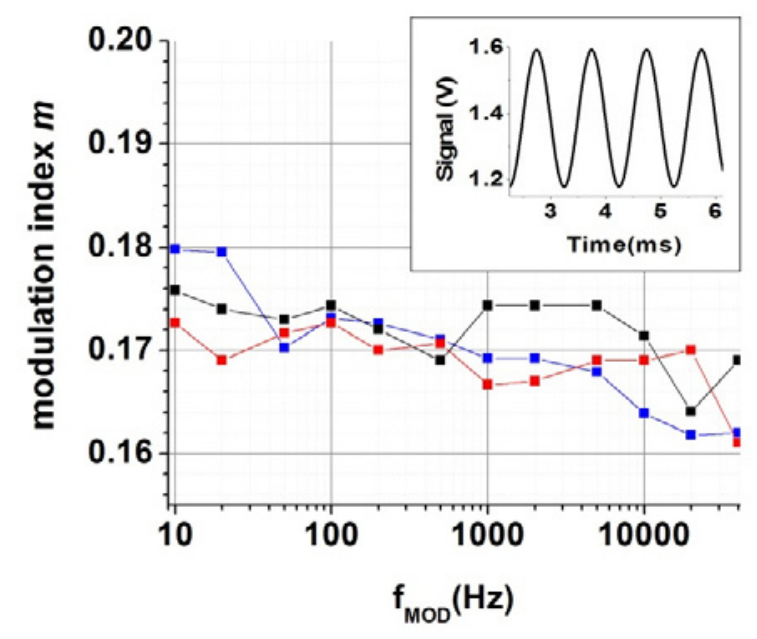

Figure 10. Intensity amplitude modulation index measured as a function of modulation frequency. Black: before the cell, blue: after the cell at resonance, red: After the cell out of resonance.

Using a setup like Sol2 in Fig.8, one can measure the RIN before the cell and use it to normalize the cell output signal by the input laser intensity. Such a system, already implemented on other compact clock setups [17] has been also tested and compared with the first solution. An equivalent RIN reduced to $7 \times 10^{-6}$ can be obtained, giving a $1.5 \times 10^{-13}$ fractional frequency stability contribution.

In order to confirm the RIN contribution calculations, the clock has been operated in a regime where the RIN noise clearly dominates the noise budget. To do so, white noise has been injected on the total laser intensity through the RF power of the switching AOM, see Fig. 4. The corresponding frequency stability has been measured and calculated with or without the intensity normalization, see Fig. 11 bottom.

Calculation and measurements are in good agreement regarding the free laser intensity case, showing that RIN is the limiting parameter when it is not reduced. In the normalized case, calculations give a lower estimation than measurements; it underlines the presence of an other noise source. 


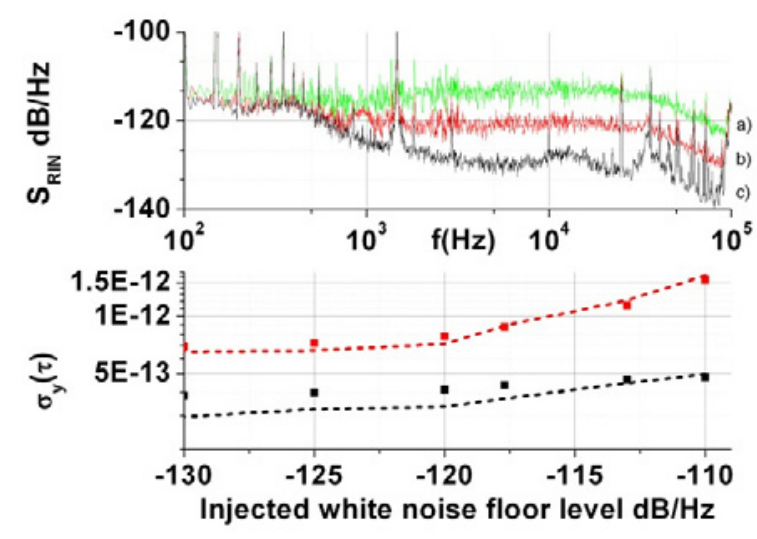

Figure 11. Top: Power spectral density of the RIN in $\mathrm{dB} / \mathrm{Hz}$. Injected noise floor of $-112 \mathrm{~dB} / \mathrm{Hz},-120 \mathrm{~dB} / \mathrm{Hz}$, lower than -140 $\mathrm{dB} / \mathrm{Hz}$ for $\mathrm{a}, \mathrm{b}$ and $\mathrm{c}$ respectively. Bottom: fractional frequency stability as a function of injected noise floor. Red upper curve: without normalization, Black lower curve: with normalization.

To conclude, the RIN reduction is fundamental to reach stability beyond $5 \times 10^{-13}$. The normalization process, which only numerically suppresses the noise, is more efficient that the power lock and would contribute with our working parameters to a fractional frequency stability of about $1.5 \times 10^{-13}$ compared to $1.9 \times 10^{-13}$ for the power lock case. The stability presented in part 4.4 has been measured in power lock mode, giving a good hope to improve it again by using the normalization mode.

\subsubsection{Local oscillator noise}

In clock working mode, the pumping duration is devoted to build the CPT state and the free evolution time is dedicated to compare the LO frequency and the atomic frequency. During such a comparison the pumping duration is equivalent to a dead time where the LO noise will not be probed and then not corrected. From this lack of information arises the so-called Dick effect [18] which describes how the clock frequency will be noised due to this sampled comparison. In order to calculate it, the sensitivity function $g(t)$ of the clock to a phase step of the LO signal must be first evaluated as $[18,19]$ :

$$
g(t)=\lim _{\Delta \varphi \rightarrow 0} \delta S(t, \Delta \varphi) / \Delta \varphi
$$

$g(t)$ has been calculated and measured on our setup, see Fig. 12, both are in good agreement.

As a second step the contribution of Dick effect to the fractional frequency stability is evaluated with [19]:

$$
\sigma_{y}^{2}(\tau)=\frac{1}{\tau} \sum_{m=1}^{\infty}\left[\frac{g_{m}^{2}}{g_{0}^{2}} S_{y}^{f}\left(\frac{m}{T_{c}}\right)\right],
$$

Where $\sigma_{y}^{2}(\tau)$ is the fractional Allan variance for an averaging time $\tau, S_{y}^{f}\left(m / T_{C}\right)$ is the one-sided power spectral density (PSD) of the relative frequency fluctuations of the free running interrogation oscillator at
Fourier frequency $m / T_{\mathrm{c}}$, i.e. the harmonic frequencies of the operation frequency.

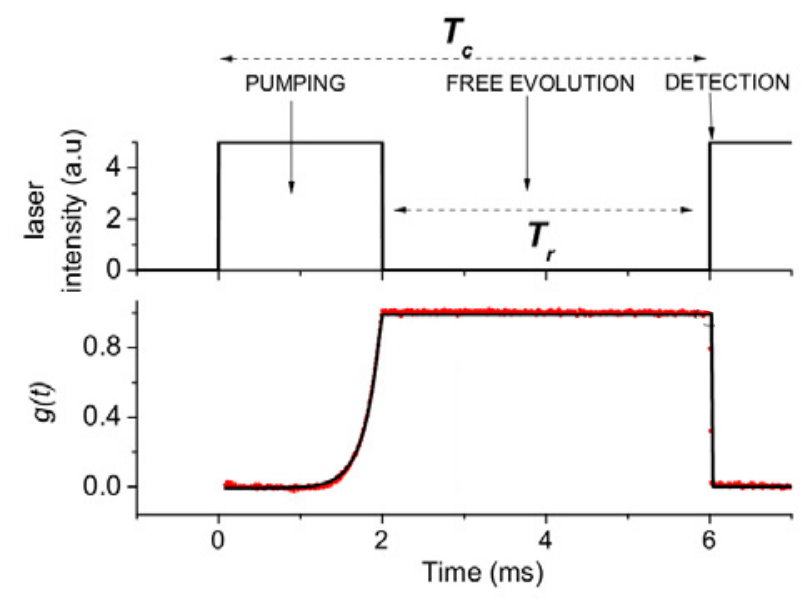

Figure 12. Top: Sequence used, pumping time: $2 \mathrm{~ms}$, Ramsey time: $4 \mathrm{~ms}$, detection $10 \mu \mathrm{s}$ after turn on of lasers, detection duration: $25 \mu \mathrm{s}$. Laser intensity: $200 \mu \mathrm{W} / \mathrm{cm}^{2} /$ laser. Bottom: sensitivity function for CPT system. Black curve: Numerical calculation, Red curve: measurements.

The parameters $g_{m}$ and $g_{0}$ are defined from the sensitivity function $g$ as follows [18]:

$$
\begin{gathered}
g_{0}=\frac{1}{T_{c}} \int_{0}^{T_{c}} g(\theta) d \theta, \\
g_{m}^{2}=\left(g_{m}^{s}\right)^{2}+\left(g_{m}^{c}\right)^{2}, \\
\left(\begin{array}{l}
g_{m}^{s} \\
g_{m}^{c}
\end{array}\right)=\frac{1}{T_{c}} \int_{0}^{T_{c}}\left(\begin{array}{l}
\sin \left(2 \pi m \theta / T_{c}\right) \\
\cos \left(2 \pi m \theta / T_{c}\right)
\end{array}\right) g(\theta) d \theta .
\end{gathered}
$$

Using this formalism, the contribution to the Dick effect of each elements of the microwave chain has been evaluated. It allowed us to choose the appropriate $100 \mathrm{MHz}$ quartz oscillator, a LNO $100 \mathrm{MHz}$ from Rakon company. The fractional frequency stability has finally been evaluated at the level of $2.7 \times 10^{-13}$ at $1 \mathrm{~s}$.

\subsection{Frequency stability}

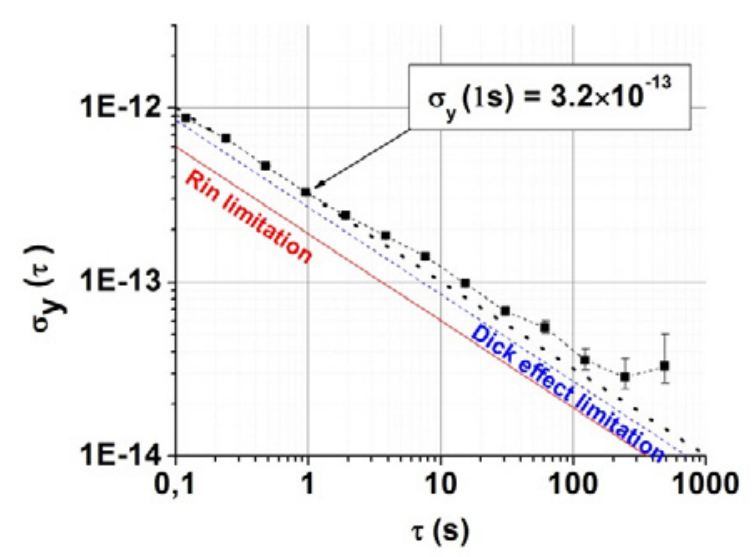

Figure 13. Short term frequency stability. Black dots: Measurements. Black dashed lines : Asymptotic behaviour of the mesurements: $3.2 \times 10^{-13} \tau^{-1 / 2}$. Red line: calculation of relative 
intensity noise contribution, blue line: calculation of LO noise contribution.

The short term fractional frequency stability has been measured at the level of $3.2 \times 10^{-13}$ using a power lock to reduce the RIN and an adapted LO quartz oscillator to reduce the Dick effect. Calculation of the RIN and Dick effects gives respectively $1.9 \times 10^{-13}$ and $2.7 \times 10^{-13}$ contributions, which combines in an overall $3.0 \times 10^{-13}$ value. The agreement with the measured value can be considered as satisfactory.

\section{Conclusion}

We have presented here a pulsed CPT vapor cell clock prototype. Firstly the quantum interfering process which allows CPT creation has been presented and its interest in term of clock performance and compactness have been developed. In a second phase, the experimental setup, the choices of optical schemes and of a pulsed mode have been detailed. Finally the studies of two mains frequency noise sources are reported: laser intensity noise and LO noise. The first study has shown that comparison between the calculated and measured contribution of the RIN to the clock stability are in good agreement. Two techniques to reduce the RIN contribution have been compared: power lock and intensity normalization. Using the power lock technique the RIN contribution to the short term frequency stability has finally been estimated at $1.9 \times 10^{-13}$ at $1 \mathrm{~s}$ integration. Regarding the LO noise, the sensitivity function calculations and measurements have allowed to estimate a Dick effect contribution at the level of $2.7 \times 10^{-}$ 13. A measurement of the short term stability has confirmed those estimations by showing a $3.2 \times 10^{-13}$ level at $1 \mathrm{~s}$, close to the $3.0 \times 10^{-13}$ estimation. This stability is at present time the best obtained on a clock based on coherent population trapping. It shows the great potential of this kind of clock.

\section{Acknowledgment}

We are grateful to M. Lours, J. Pinto and L. Volodimer for their technical assistance and the realization of various electronic devices. We are pleased to acknowledge Pierre Bonnay and Annie Gérard for manufacturing Cs cells. We are also thankful to Rodolphe Boudot for fruitful discussions.

\section{References}

1. P. Waller, F. Gonzalez, S. Binda, I. Sesia, I. Hidalgo, G. Tobias, and P. Tavella, Trans. Ultrason., Ferroelect., Freq. Contr., 57, 738, 2010.

2. G. Alzetta, A. Gozzini, L. Moi, and G. Orriols, Il Nuovo Cimento, 36, 5 (1976)

3. E. Arimondo, Progress in Optics, vol. XXXV, pp. 257-353 (1996)

4. R. Wynands, and A. Nagel, Applied Physics B, 68, 1 (1999)

5. J. Vanier, Applied Physics B, 81, 421 (2005)

6. V. Shah and J. Kitching, Advances in Atomic, Molecular, and Optical Physics, 59, 21 (2010)
7. S. Knappe, Comprehensive Microsystems, 3, 571 (2010)

8. R. Boudot., X. Liu, P. Abbé, R. Chutani, N. Passilly, S. Galliou, C. Gorecki, and V. Giordano, IEEE Trans. Ultrason., Ferroelect., Freq. Contr., 59, 2584 (2012)

9. J. Vanier, and C. Audoin, The quantum physics of atomic frequency standards, Adam Hilger, Bristol (1989).

10. N. F. Ramsey, Phys. Rev., 78, 695 (1950)

11. T. Zanon, S. Guérandel, E. de Clercq, D. Holleville, N. Dimarcq, and A. Clairon, Phys. Rev. Lett., 94, 193002 (2005)

12. R. Boudot, S. Guérandel, E. de Clercq, IEEE Trans. Instr. Meas., 58, 3659 (2009)

13. S. Guérandel, T. Zanon, N. Castagna, F. Dahes, E. de Clercq, N. Dimarcq, and A. Clairon, IEEE Trans. Instr. Meas., 56, .383 (2007)

14. X. Liu, J-M. Mérolla, S. Guérandel, E. de Clercq, and R. Boudot, Opt. Expr., 21, 12451 (2013)

15. D. Miletic, C. Affolderbach, M. Hasegawa, R. Boudot, C. Gorecki, G. Mileti, Appl. Phys. B, 109, 89 (2012)

16. L. Duchayne, PhD Thesis, Observatoire de Paris, Paris (2008)

17. F. X. Esnault, PhD Thesis, Université Pierre et Marie Curie, Paris (2009)

18. G. J Dick, in Proc. 19th Precise Time and Time Interval (PTTI) Applications and Planning Meeting, Redondo Beach, CA, USA, 133 (1987)

19. C. Audoin, G. Santarelli, Ala'a Makdissi, and André Clairon, IEEE Trans. Ultrason., Ferroelect., Freq. Contr., 45, 877 (1998) 